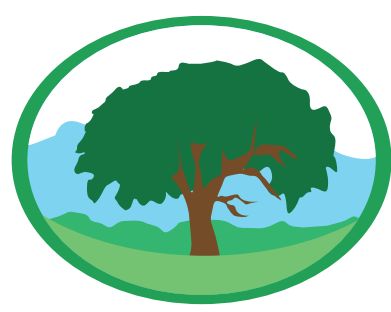

\title{
PROPRIEDADES TÉRMICAS DE COMPÓSITOS DE POLIETILENO DE ALTA DENSIDADE REFORCุADOS COM CELULOSE
}

\author{
BOSENBECKER, M. W. ${ }^{1}$; CROCHE, S. ${ }^{2}$; CHOLANT, G. ${ }^{2}$; ROSA, P. ${ }^{2}$; PASSADOR, F. ${ }^{3}$; MARINI, J. ${ }^{3}$; OLIVEIRA, A. ${ }^{4}$. \\ ${ }^{1}$ Universidade Federal de Pelotas \\ ${ }^{2}$ Universidade Federal de Pelotas - Centro de Desenvolvimento Tecnológico/CDTec - Pelotas/RS \\ ${ }^{3}$ Instituto de Ciência e Tecnologia (ICT) - Engenharia de Materiais - Universidade Federal de São Paulo (UNIFESP), São José \\ dos Campos/SP
}

${ }^{4}$ Universidade Federal de Pelotas - Programa de Pós Graduação em Ciência e Engenharia de Materiais/PPGCEM - Pelotas/RS

\section{Palavras-chave: celulose, Resumo}

polietileno de alta densidade, Este trabalho buscou desenvolver compósitos utilizando como matriz polimérica casca de arroz, compósitos o polietileno de alta densidade e como reforço foi utilizada a celulose obtida a partir da casca de arroz. Os compósitos obtidos foram preparados nas proporções de 5,10 e $15 \%$ em massa de celulose, utilizando-se para isto uma extrusora de rosca simples. A celulose obtida foi caracterizada por difração de raios X. Os materiais compósitos foram caracterizados através das técnicas de análise termogravimétrica e calorimetria exploratória diferencial. Por meio dos resultados de difração de raios $X$ foi possível comprovar um aumento do índice de cristalinidade da celulose após sucessivos tratamentos químicos, assim como ligeiro aumento na estabilidade térmica com a adição de carga. No entanto, os dados obtidos por calorimetria exploratória diferencial mostraram que aparentemente a celulose não apresenta influência significativa nas temperaturas de transição do polímero.

\section{THERMAL PROPERTIES OF HIGH-DENSITY POLYETHYLENE COMPOSITES REINFORCED WITH CELLULOSE}

\section{Keywords:}

high density

rice husk. cellulose.

polyethylene. composites

\begin{abstract}
This study sought to develop composites using polymeric matrix the high-density polyethylene and cellulose was used as reinforcement obtained from rice husk. The composites were prepared in the proportions of 5,10 and $15 \%$ by mass of cellulose, using for this a single screw extruder. The cellulose pulp obtained was characterized by $x$-ray diffraction. Composite materials were characterized by thermogravimetric analysis and differential scanning calorimetry. Through the results of $x$-ray diffraction it was possible to demonstrate an increase in the index of crystallinity of cellulose after successive chemical treatments, as well as a slight increase in thermal stability with the addition of load. However, the data obtained by differential scanning calorimetry did not show a significant improvement in their results.
\end{abstract}




\section{INTRODUÇÃO}

A crescente demanda por materiais de fontes renováveis com propriedades diferenciadas tem possibilitado a inserçáo de produtos e resíduos da agricultura (agroindustriais) em diferentes áreas de pesquisa. $\mathrm{O}$ aproveitamento de resíduos da atividade agrícola na produção de compósitos e nanocompósitos poliméricos,pode reduzir os custos do produto final, além de promover um destino adequado a estes resíduos, agregando-lhes valor (SIQUEIRA et al., 2010).

Neste sentido, estudos têm demonstrado interesse na incorporação de fibras vegetais em matrizes poliméricas, proporcionando melhoria de suas propriedades e por substituírem, geralmente com vantagens econômicas e de densidade, os convencionais reforços inorgânicos como as fibras de vidro (PÖLLÄNEN, 2013).

A casca de arroz (CA) é um resíduo agroindustrial produzido em grandes quantidades no Rio Grande do Sul (IBGE, 2018). Estas cascas possuem baixo valor comercial, pois a sílica e as fibras contidas não possuem valor nutritivo e por isso não são usadas na alimentação humana ou animal, resultando em sérios impactos ambientais. Mais recentemente este material tem sido empregado também como reforço em materiais compósitos poliméricos. A CA caracteriza-se também por ser considerada como a fonte mais abundante de celulose a partir de resíduos agroindustriais do mundo (ROSA et al., 2012; JOHAR; AHMAD; DUFRESNE, 2012).

Além disso, não há trabalhos publicados na literatura sobre a utilização da celulose obtida a partir da CA em matriz de polietileno de alta densidade (PEAD) principalmente em pesquisas realizadas no Brasil) (BATTEGAZZORE et al., 2014). Neste contexto, o objetivo deste trabalho foi caracterizar a celulose e avaliar o efeito da utilização de diferentes concentraçôes de carga nas propriedades térmicas de materiais compósitos com matriz de PEAD.

\section{MATERIAL E MÉTODOS}

\section{Materiais}

O polímero utilizado neste trabalho foi o polietileno de alta densidade (PEAD), conhecido comercialmente como PEAD IG58, fornecido pela Petroquímica Braskem S/A. A casca de arroz utilizada foi doada pela empresa Cerealista Polisul, localizada na cidade de Pelotas-RS, Brasil.

Obtençáo da Celulose a partir da Casca de Arroz

A obtenção das fibras de celulose a partir da casca de arroz envolveu duas etapas: tratamento alcalino e branqueamento. $\mathrm{O}$ tratamento alcalino foi realizado com o objetivo de remover a hemicelulose e a lignina da casca.

Para isto, 35 gramas de casca na forma de um pó fino, doada pela empresa Cerealista Polisul, foi colocada em uma solução de $700 \mathrm{ml}$ de água destilada, $290 \mathrm{ml}$ de ácido acético $\left(\mathrm{CH}_{3} \mathrm{COOH}\right)$ e $60 \mathrm{ml}$ de ácido nítrico $\left(\mathrm{HNO}_{3}\right)$, esta solução foi deixada em banho-maria durante 4 horas. No término da reação, a solução foi filtrada e o material sólido obtido foi lavado várias vezes até atingir um $\mathrm{pH}$ neutro.

Após tratamento alcalino, o processo de branqueamento foi realizado com adição da casca tratada em solução de $700 \mathrm{ml}$ de hipoclorito de sódio $(\mathrm{NaClO})$ comercial durante 24 horas. O material resultante foi filtrado e também lavado com água destilada. Em seguida, foi seco em estufa de secagem da marca Quimis a $60^{\circ} \mathrm{C}$, por 24 horas.

\section{Preparaçáo dos Compósitos PEAD/Celulose}

Os compósitos foram obtidos pelo método de mistura no estado fundido, com o auxílio de uma extrusora de rosca simples com temperaturas de $180^{\circ}$, $180^{\circ}$ e $190^{\circ} \mathrm{C}$, nas zonas 1,2 e 3 , respectivamente.

\section{Difraçáo de Raios X (DRX)}

A técnica de DRX permite identificar a estrutura cristalina do material, bem como o seu grau de cristalinidade.

As análises de DRX foram realizadas utilizandose um difratômetro D8 Advance Bruker, com comprimento de onda $(\lambda=1,541 \AA)$, operando a 40 $\mathrm{KV}$ e $40 \mathrm{~mA}$. A varredura foi realizada na faixa de $2 \lambda$ entre 10 e $50^{\circ}$, a uma taxa de $1 \% / \mathrm{min}$.

Com os difratogramas obtidos foi possível determinar o índice de cristalinidade da celulose de acordo com a Eq. 1, segundo a metodologia proposta por Segal et al. (1959):

$$
\mathrm{I}_{\mathrm{c}}=\frac{\mathrm{I}(002)-\mathrm{I}(\mathrm{am})}{\mathrm{I}(002)} * 100 \%
$$


onde: $\mathrm{I}_{002}$ é o valor de intensidade máximo de difração do pico próximo a $2 \theta=22^{\circ}$, relacionado a parte cristalina do material, enquanto $\mathrm{I}_{\mathrm{am}}$ é a intensidade do halo de difração do pico de que representa o material amorfo em $2 \theta-18^{\circ}$ (SEGAL et al., 1959).

\section{Análise Termogravimétrica (TGA)}

A análise termogravimétrica tem como objetivo avaliar a estabilidade térmica do material estudado. Para esta análise foi utilizado um equipamento da TA Instruments, modelo TGA Q50, a uma taxa de $20^{\circ} \mathrm{C} /$ min, a partir da temperatura ambiente até $700^{\circ} \mathrm{C}$, sob atmosfera de nitrogênio.

\section{Calorimetria Exploratória Diferencial (DSC) do}

\section{Compósito}

A calorimetria exploratória diferencial (DSC) foi realizada para avaliar o efeito da celulose nas temperaturas de fusão $\left(\mathrm{T}_{\mathrm{m}}\right)$, de cristalização $\left(\mathrm{T}_{\mathrm{c}}\right)$, e no grau de cristalinidade do polímero. Foi utilizado um equipamento da TA Instruments, modelo Q-1000, com nitrogênio como gás de arraste, em fluxo constante de $50 \mathrm{ml} / \mathrm{min}$ (ASTM E1269 - 11).

As amostras foram inicialmente aquecidas da temperatura ambiente até $200{ }^{\circ} \mathrm{C}$ a uma taxa de aquecimento de $10 \mathrm{\circ} \mathrm{C} / \mathrm{min}$, permanecendo nesta temperatura por 3 minutos. Logo após foram resfriadas até $30^{\circ} \mathrm{C}$ a uma taxa de $10{ }^{\circ} \mathrm{C} / \mathrm{min}$ para a determinaçáo da temperatura de cristalização $\left(T_{c}\right)$ e novamente foram aquecidas até $200{ }^{\circ} \mathrm{C}$ a uma taxa de $10^{\circ} \mathrm{C} / \mathrm{min}$. Para a realização das análises foram utilizados os termogramas do segundo aquecimento por não possuírem influência da história térmica das amostras.

Através da Eq. 2 foi determinado o grau de cristalinidade $\left(\mathrm{X}_{\mathrm{c}}\right)$ dos materiais estudados (CANEVAROLO, 2006):

$$
\mathrm{X}_{\mathrm{c}}=\frac{\Delta \mathrm{Hm}}{\Delta \mathrm{H}_{0}(1-\varnothing)} * 100 \%
$$

Eq. (2)

\section{RESULTADOS E DISCUSSÃO}

\section{O Difraçáo de Raios X (DRX)}

A difratometria de raios $\mathrm{X}$ foi utilizada para investigar a estrutura cristalina da casca de arroz antes e após os tratamentos químicos. A celulose é de natureza cristalina devido a interaçôes de ligaçóes de hidrogênio e forças de Van der Waals entre moléculas adjacentes contrárias à hemicelulose e à lignina, que possuem uma estrutura amorfa (PRADO; SPINACÉA, 2015).

Os difratogramas de raios $\mathrm{X}$ das amostras obtidas estão apresentados na Figura 1, no qual podemos observar a presença de três picos característicos de celulose tipo I, em aproximadamente $16^{\circ}, 22^{\circ}$ e $34^{\circ}$ correspondentes aos planos (101), (002), (040), respectivamente (HALAL et al., 2015).

$64,12 \%$

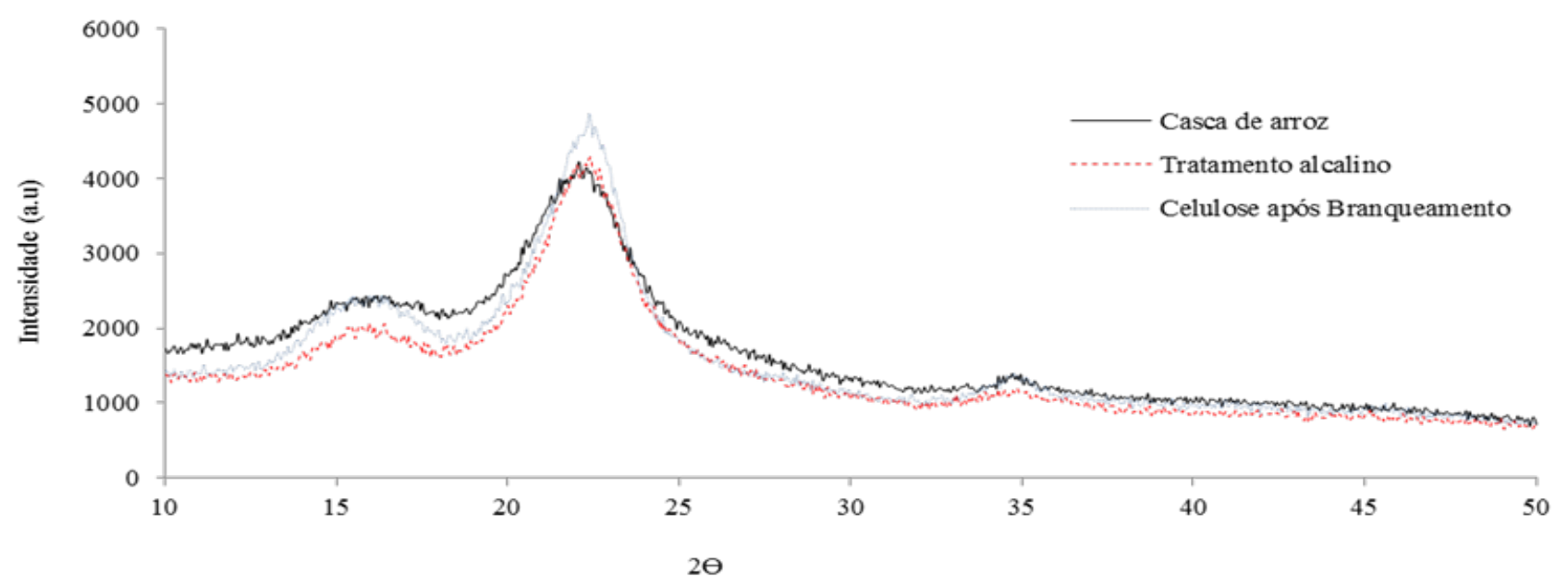

Figura 1. Difratograma de raios-x das amostras obtidas

$\mathrm{Na}$ Tabela 1, estáo apresentados os resultados para cristalinidade obtidos a partir do método de Segal et al (1959). Pode-se notar o aumento da cristalinidade para a celulose obtida após branqueamento em relaçáo à casca 
de arroz, a qual apresentou um Ic de 64,12\%, este resultado é devido à remoção de materiais não celulósicos (BETTAIEBA et al., 2015).

Tabela 1.Índice cristalinidade (\% IC) calculado através do método de Segal

\begin{tabular}{llll}
\hline Amostras & Casca de Arroz & Tratamento Alcalino & Branqueamento \\
\hline$\% \mathrm{Cr}$ & 47,80 & 58,65 & 64,12 \\
\hline
\end{tabular}

\section{Análise Termogravimétrica (TGA)}

Os termogramas de TGA da celulose e dos compósitos com 5, 10 e 15\% de celulose são relatados

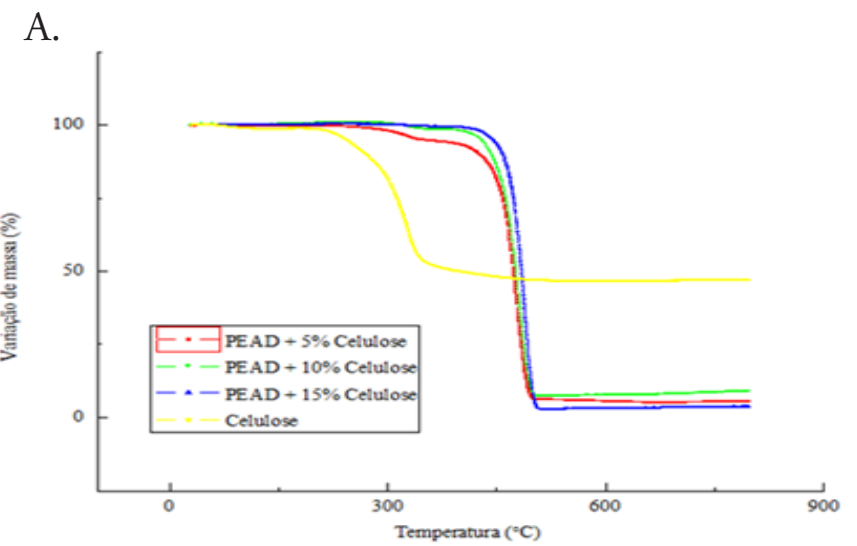

na Figura 2A enquanto na Figura 2B a derivada das curvas de perda de massa (DTG) é apresentada.

Segundo a literatura, a decomposição térmica das fibras naturais ocorre em diferentes temperaturas. A decomposição da hemicelulose inicia-se por volta de $220^{\circ} \mathrm{C}$ e continua até os $300^{\circ} \mathrm{C}$, a celulose tem sua degradação iniciada perto dos $310^{\circ} \mathrm{C}$ até os $400^{\circ} \mathrm{C}$, enquanto a lignina apresenta temperaturas de degradação entre $200^{\circ} \mathrm{C}$ até $700^{\circ} \mathrm{C}$ (ABRAHAM et al., 2011).

Através da análise da curva de TGA para a celulose é possível observar dois processos de decomposição.

B.

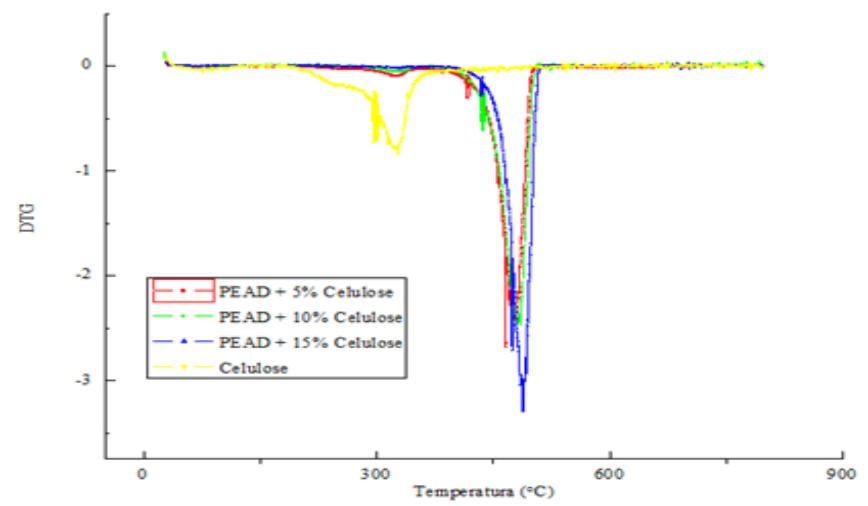

Figura 2. A. Curvas de TGA e B. Curvas de DTG da celulose e dos compósitos estudados.

$\mathrm{O}$ primeiro processo refere-se à uma perda de massa inicial em torno de $100^{\circ} \mathrm{C}$, ficando em aproximadamente em $2 \%$ para a celulose, que foi devido à desidratação do material, que, mesmo tendo sido seco antes das análises, apresenta caráter hidrofílico, dificultando a total eliminação de água. A segunda perda de massa ocorreu entre 200 e $400^{\circ} \mathrm{C}$ e foi atribuído à degradação da hemicelulose, pectina, lignina e celulose (ROSA et al., 2010).

Analisando os resultados obtidos para os materiais compósitos podemos notar que há um pequeno aumento da estabilidade térmica do compósito com o aumento do teor de carga (ROSA, et al 2010).

O resultado deste trabalho está de acordo como reportado por Johar e Ahamad (2012), onde o compósito praticamente não perdeu peso em $400^{\circ} \mathrm{C}$; contudo, a partir de $450{ }^{\circ} \mathrm{C}$, a perda de peso foi rápida e acentuada, resultando em resíduos mínimos.

A Figura 2B mostra a curva da primeira derivada da perda de massa (DTG), onde é observado um primeiro pico em aproximadamente $327^{\circ} \mathrm{C}$ para a celulose, referente à máxima taxa de decomposição da celulose. Um segundo pico é evidenciado em aproximadamente $487^{\circ} \mathrm{C}$ e que equivale à máxima taxa de decomposição do PEAD, onde observa-se uma sobreposição dos picos, indicando que a adição de celulose na matriz polimérica contribui para a obtenção de compósitos sem gerar perdas na estabilidade térmica (BORSOI et al., 2011).

\section{Calorimetria Exploratória Diferencial (DSC)}

$\mathrm{Na}$ Figura 3A e B encontram-se os termogramas de DSC do resfriamento e do segundo aquecimento, respectivamente, que foram utilizados para caracterizar o PEAD e os compósitos com 5, 10 e 15\% em massa de celulose. Os valores correspondentes a temperatura de fusão cristalina $(\mathrm{Tm})$, entalpia de fusão $(\Delta \mathrm{Hm})$, temperatura de cristalização (Tc), entalpia de cristalização $(\Delta \mathrm{Hc})$, estâo demonstrados na Tabela 2. 
A.

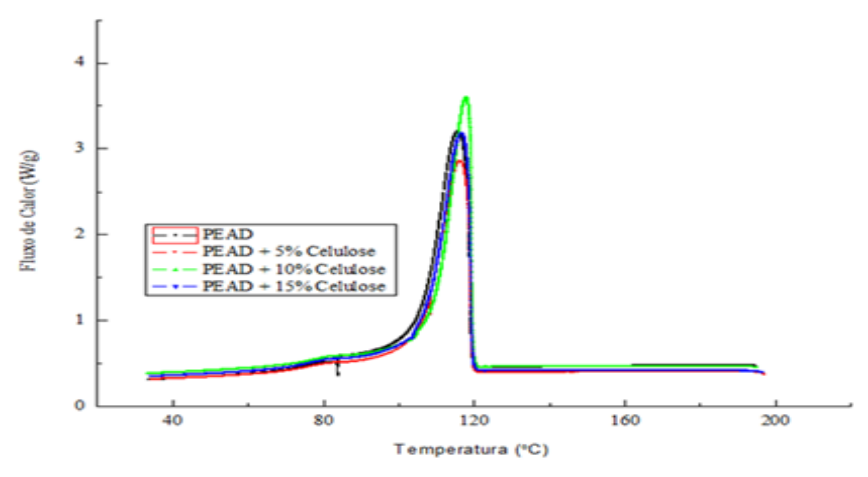

B.

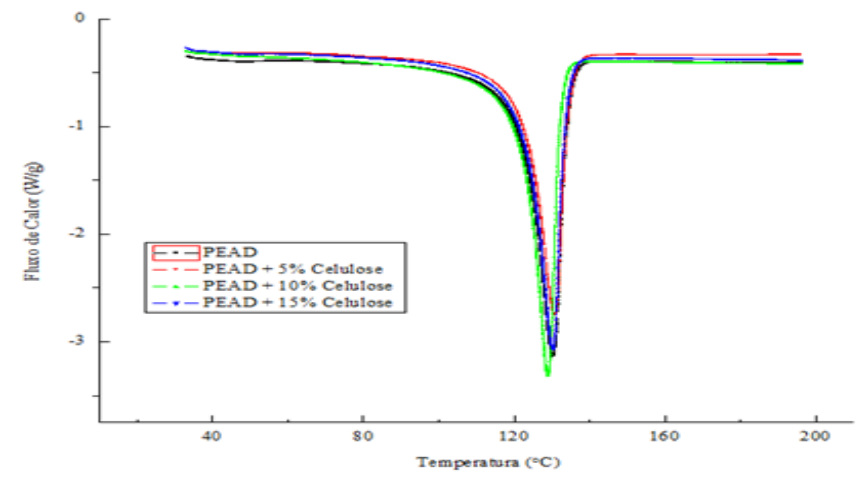

Figura 3. A - B. Termogramas de DSC dos materiais estudados referente ao resfriamento e segundo aquecimento, respectivamente.

Tabela 2. Valores obtidos por DSC da temperatura de cristalização $\left(T_{c}\right)$, temperatura de fusão $\left(T_{f}\right)$, entalpia de cristalização $\left(\Delta \mathrm{H}_{\mathrm{c}}\right)$, entalpia de fusão $\left(\Delta \mathrm{H}_{\mathrm{f}}\right)$ e grau de cristalinidade $\left(\% X_{c}\right)$.

\begin{tabular}{cccccc}
\hline Amostra & $\mathrm{T}_{\mathrm{c}}\left({ }^{\circ} \mathrm{C}\right)$ & $\mathrm{T}_{\mathrm{m}}\left({ }^{\circ} \mathrm{C}\right)$ & $\left.\Delta \mathrm{H}_{\mathrm{c}} \mathrm{J} / \mathrm{g}\right)$ & $\Delta \mathrm{H}_{\mathrm{m}}(\mathrm{J} / \mathrm{g})$ & $\mathrm{X}_{\mathrm{c}}(\%)$ \\
\hline PEAD & 115,52 & 132,54 & 164,50 & 182,20 & 62,18 \\
$\mathrm{PEAD}+5 \%$ & 116,82 & 132,61 & 99,04 & 134,20 & 45,80 \\
$\quad$ Celulose & & & & & \\
$\begin{array}{c}\text { PEAD + 10\% } \\
\text { Celulose }\end{array}$ & 117,90 & 131,06 & 140,20 & 158,40 & 54,06 \\
$\begin{array}{c}\text { PEAD + 15\% } \\
\text { Celulose }\end{array}$ & 116,63 & 132,01 & 138,60 & 160,60 & 54,81 \\
\hline
\end{tabular}

Analisando a Tabela 2 é possível observar que os valores de temperaturas de fusão e de cristalização dos materiais compósitos foram similares aos valores obtidos para o PEAD puro. (BORAN et al., 2016). Resultados semelhantes foram obtidos por Fernandes (2015), que estudou materiais compósitos com matriz de epóxi reforçada com cinza de casca de arroz. O autor observou que as curvas de DSC dos compósitos e da matriz são muito similares, indicando que a presença de diferentes concentraçôes de cinza não alterou significativamente o comportamento térmico do polímero (FERNANDES, 2015).

Analisando as entalpias de fusão e cristalização, percebe-se um aumento dos valores de entalpia com o aumento do teor de celulose, porém com uma pequena reduçáo para o compósito de $15 \%$ de celulose e quando comparado com o PEAD puro, observa-se uma diminuiçãao bastante pronunciada em relação aos compósitos estudados.

Os valores de grau de cristalinidade para os compósitos foram calculados usando a cristalinidade do PEAD virgem ( $\triangle \mathrm{H} 100 \%)$ como parâmetro, 293 $\mathrm{KJ} / \mathrm{mol}$. O grau de cristalinidade encontrado neste trabalho $(62,18 \%)$ está de acordo com o relatado na literatura, onde a faixa do grau de cristalinidade para o PEAD está na faixa de 62 e 82\% (BECERRA, 2015).

Todos os compósitos apresentaram um grau de cristalinidade menor que o PEAD, que pode ser um indício de que as cargas utilizadas não atuaram como agentes nucleantes, que aumentariam a cristalinidade do compósito conforme relatado na literatura (GOMES; VISCONDE; PACHECO, 2013).

\section{CONCLUSÃO}

O método utilizado para a obtenção da celulose a partir da casca de arroz foi eficiente, revelando um aumento da cristalinidade após tratamentos químicos. Verificou-se um ligeiro aumento na estabilidade térmica com a adição de carga a matriz polimérica, no entanto, não foi observado uma mudança significativa nas temperaturas de fusão e de cristalização para as amostras estudadas. Vale ressaltar que o reaproveitamento deste resíduo contribui para a diminuição do impacto ambiental e reutilização deste material.

\section{AGRADECIMENTOS}

Ao Laboratório de Materiais Compósitos (LABCOM - UFPEL) pelo espaço físico disponibilizado para a realização da parte experimental. À Petroquímica Braskem pela doação do polietileno de alta densidade e a Cerealista Polisul pela doação da casca de arroz. Ao Centro de Microscopia Eletrônica 
do Sul (CEME-SUL-FURG) pela a realização da análise de difração de raios $\mathrm{X}$.

\section{LITERATURA CITADA}

ABRAHAM, E., DEEPA, B.; POTHAN, L. A.; JACOB, M.; THOMAS, S.; CVELBAR, U.; ANANDJIWALA, R.E. Extraction of nanocellulose fibrils from lignocellulosic fibres: A novel approach. Carbohydrates Polymers, v. 86, n. 4, p. 14681475, 2011.

ASTM E1269 - 11 - Standard test method for determining specific heat capacity by differential scanning calorimetry.

BATTEGAZZORE, D.; BOCCHINI, S.; ALONGI, J.; FRACHE, A.; MARINO. Cellulose extracted from rice husk as filler for poly (lactic acid): preparation and characterization. Springer Science Business Media Dordrecht, v. 21, p. 18131821, 2014.

BECERRA, A. F. C. Caracterização de PEAD antes e após envelhecimento em diferentes meios e condiçóes. Dissertação Mestrado - Pontifícia Universidade Católica do Rio de janeiro, Departamento de Engenharia Química e de Materiais, 2015.

BETTAIEBA, F; KHIARI, R.; HASSAN, M. L.; BELGACEM, M. N.; BRAS, J.; DUFRESNE, A.; MHENNI, M. F. Preparation and characterization of new cellulose nanocrystals from marine biomass Posidonia oceanica. Industrial Crops and Products, v. 72, p. 175-182, 2015.

BORAN, S.; Kiziltas, A.; Kiziltas, E. E.; Gardner, D. J. The comparative study of different mixing methods for microcrystalline cellulose/polyethylene composites. International Polymer Processing, v. 31, n. 1, p. 92-103, 2016.

BORSOI, Cleide.; SCIENZA, Lisete C.; ZATTERA, Ademir J.; ANGRIZANI, Clarissa C. Obtenção e caracterização de compósitos utilizando poliestireno como matriz e resíduos de fibras de algodão da indústria têxtil como reforço. Polímeros: Ciência e Tecnologia, v. 21, n. 4, p. 271-279, 2011.

CANEVAROLO, S. V. Ciência dos Polímeros. Um curso básico para tecnólogos e engenheiros. 2. Ed. São Paulo: Artiliber, v. 1, p. 289, 2006.

FERNANDES, I. J. Desenvolvimento e caracterização de compósitos de cinza de casca de arroz em matriz epóxi. Programa de Pós-Graduação em Engenharia Civil, Sáo Leopoldo, 2015.

GOMES, T. S.; VISCONDE, L. L. Y.; PACHECO, E.B.A.V. Substituição da fibra de vidro por fibra de bananeira em compósitos de polietileno de alta densidade. Parte 1. Avaliação mecânica e térmica. Polímeros: Ciência e Tecnologia, v. 23, p. 206-211, 2013.

HALAL, S.L.M.; COLUSSI, R.; DEON, V. G.; PINTO, V. Z.; VILLANOVA, F. A.; CARREÑO, N. L. V.; ZAVAREZE, E.
DA R. Films based on oxidized starch and cellulose from barley. Carbohydrates Polymers, v. 133, p. 644-653, 2015.

JOHAR, N.; AHMAD, I. Morphological, thermal, and mechanical properties of starch biocomposite films reinforced by cellulose nanocrystals from rice rusks. BioResources, v. 7, n. 4, p. 5469-5477, 2012.

JOHAR, N.; AHMAD, I.; DUFRESNE, A. Extraction, preparation and characterization of cellulose fibres and nanocrystals from rice husk. Industrial Crops and Products, v. 37, p. 93- 99, 2012.

PÖLLÄNEN, M.; SUVANTO, M.; PAKKANEN, T.T. Cellulose reinforced high density polyethylene composites - Morphology, mechanical and thermal expansion properties. Composites Science and Technology, v. 76, p. 21-28, 2013.

PRADO, K.S.; SPINACÉA, M.A.S. Characterization of fibers from pineapple's crown, rice husks and cotton textile residues. Materials Research, v.18, n.3, p. 530-537, 2015.

ROSA, M. F.; MEDEIROS, E. S.; MALMONGE, J. A.; GREGORSKI, K. S.; WOOD, D. F.; MATTOSO, L. H. C.; IMAM, S. H. Cellulose nanowhiskers from coconut husk fibers: Effect of preparation conditions on their thermal and morphological behavior. Carbohydrates Polymers, v. 81, n. 1, p. 83-92, 2010.

ROSA, S. M. L.; REHMAN, N.; DE MIRANDA, M. I. G.; NACHTIGALL, S. M. B.; BICA, C. I. D. Chlorine-free extraction of cellulose from rice husk and whisker isolation. Carbohydrate Polymers, v. 87, p.1131-1138, 2012.

RS é o maior produtor de arroz do país, aponta IBGE | Brasil 247. Disponível em: <https://www.brasil247.com/.../RS-é-omaior-produtor-de-arroz-do-país-aponta-IBGE> Acesso em 08 jul. 2018.

SEGAL, L.; CREELY, J. J.; MARTIN, A. E.; CONRAD, C. M. An empirical method for estimating the degree of crystallinity of native cellulose using the $\mathrm{x}$-ray diffractometer. Textile research journal, v. 29, n. 10, p. 786-794, 1959.

SIQUEIRA, G.; TAPIN-LINGUA, S.; PEREZ, D. da S.; DUFRESNE, AL. Morphological investigation of nanoparticles obtained from combined mechanical shearing, and enzimatic and acid hydrolysis of sisal fibers. Cellulose, v. 17, n. 6, p. 1147$1158,2010$. 